\title{
Towards Superluminal Physics: Compromising Einstein's Special Relativity and Faster-Than-Light Particles
}

\author{
Lukasz Andrzej Glinka* \\ B.M. Birla Science Centre, Hyderabad, India \\ *Corresponding author: laglinka@gmail.com; lukaszglinka@wp.eu
}

Received May 24, 2014; Revised June 02, 2014; Accepted June 09, 2014

\begin{abstract}
Throughout the violation of momentum-velocity parallelism and deformation of the Einstein equivalence principle, the model of faster- than-light motion, wherein both the Minkowski energy-momentum space and the Lorentz invariance, laying the foundations of Special Relativity and Standard Model, is constructed. Recently announced and denounced CERN's superluminal neutrinos are confronted.
\end{abstract}

Keywords: special relativity, deformed relativity, faster-than-light, particles, superluminal motion, neutrino, Lorentz symmetry

Cite This Article: Lukasz Andrzej Glinka, "Towards Superluminal Physics: Compromising Einstein's Special Relativity and Faster-Than-Light Particles.” Applied Mathematics and Physics, vol. 2, no. 3 (2014): 94-102. doi: 10.12691/amp-2-3-5.

\section{Introduction}

On September 22, 2011, the human world overloaded throughout the diverse paradigms and dogmas had experienced truly revolutionary excitations. Namely, on this day the OPERA Collaboration, an international experimental project of the European Organization for Nuclear Research - CERN, announced that their results, which arose from the high-statistics experimental data, evidently demonstrate existence of the superluminal neutrinos, Cf. the Refs. [1,2]. During the subsequent five months, the public opinion was a witness of many various speculations about faster-then-light motion, but already on February 22, 2012, OPERA pointed out the two issues, based on the technology of the Global Positioning System whose construction in itself makes use of the arguments of Special Relativity, which could immediately impact on the measurement process, Cf. the Refs. [3,4]. The first problem was linked to the oscillator producing the events time-stamps in between the GPS synchronizations, whereas the second one was the cable of the optical fiber bringing the external GPS signal to the OPERA master clock. Both these possibilities potentially could give the anomaly regarded as an experimental error which led to registration of the faster-than-light neutrino. In February 2012, the second reason was considered more seriously than the first one, and the CERN experimentalists announced that the situation will be verified once again yet in 2012. In March 2012, the ICARUS experiment, another CERN experimental collaboration initiated in 1977 by Carlo Rubbia, who shared the 1984 Nobel Prize in Physics for discovery of the weak gauge bosons $\mathrm{W}$ and
$\mathrm{Z}$, conirmed the absolutism of the speed of light in the measurement of the neutrino motion, Cf. the Ref. [5]. Already in June 2012, the CERN Research Director Sergio Bertolucci, at the 25th International Conference on Neutrino Physics and Astrophysics held in Kyoto, confirmed the mistaken measurement due to the OPERA Collaboration, Cf. the Ref. [6]. Since the CERN neutrinos were suspected of faster-than-light travels, Special Relativity could have problems due to the inconsistencies with superluminal motion. Strictly speaking, certainly this, potential fall of reduction in favour of pluralism, could be the major contribution to a definitive bankruptcy of particle physicists and changing the status of the Geneva sacred sanctuary from the top scientific research institution to at most an ideological museum, or even the graveyard. Moreover, such paradigm shift could relocate theoretical physics from sciences which collect the only useful endeavours which are able to make a civilization progress in both technological and living conditions, to arts whose usefulness is defined through the individual tastes and are able to at most a cultural progress, or even to religions whose aim is gaining money throughout development of ideology and hierarchy.

Nevertheless, repeating the phrase IIt is not even wrong", coined by Wolfgang Pauli who received the 1945 Nobel Prize in Physics for the discovery of the exclusion principle, this is clear that although an openly breaking character of the aforementioned state of afiairs, this event has already motivated a serious reconsideration of the possibility for the faster-than-light motion. Strictly speaking, this possibility is manifestly against the foundations of theoretical physics arising from Special Relativity, cultivated as one of the masterpieces of modern physics founded in 1905 by Albert Einstein who was 
awarded by 1921 Nobel Prize in Physics for his services to theoretical physics and discovery of the law of the photoelectric effect, and is considered as one of the fatherfounders of modern theoretical physics, which can be compared with a dogma of faith. Namely, the ideological and technical catastrophe of the OPERA Collaboration research in measurement of speed of neutrinos lead to the essential question on the limits of applicability of the Special Relativity, that is the physical boundaries of Einstein's postulate about the absolute monarchy rule of the speed of light $\mathrm{c}$ in the kingdom of speeds. Interestingly, an existence of faster-than-light particles, and their relation to Special Relativity, is not a new research trend in physics. Its most intensive development took place starting the early 1960s with a peak in 1970s, Cf. the Refs. $[7,19]$ and references therein, and also much later this subject has been discussed in different contexts, Cf. the Refs. [20,21,22] and references therein. Moreover, it is worth stressing that the superluminal state of affairs is well-known in modern astronomy since the early 1980s, when the faster-than-light motion had been suggested in order to contradict the quasars having the cosmological distances. In the present-day situation, the experimental data show that the superluminal travels are the phenomena which are very often met in radio galaxies, quasars and microquasars. Usually, this is believed that an existence of a black hole within these sources provokes the high speed mass ejection. Furthermore, it should be emphasized that the optical illusions are mostly believed to be the theoretical justification for the superluminal speeds, and such arguments do not destroy the spirit of Einstein's Special Relativity. For this reason, the speculations based on the conclusions about the hypothetical possibilities such like time travels, arising from the exceeding of the speed of light, are still propagated. Furthermore, it should be emphasized that even Albert Einstein offcially never excluded the possibility of superluminal speeds, in spite that according to Special Relativity faster-than-light particles has a purely imaginary proper mass, that is the squared mass has a negative value, what is regarded as a non-physical situation. His account of the theory of motion is the postulate of Special Relativity, which states that the speed of light $\mathrm{c}$ is the maximal velocity able to be reached by a physical object called photon, which, however, in not equivalent to the statement that the measured speed of a moving object must be less than c. Furthermore, because actually nobody knows what really happens when an object reaches the speed very close to c, most likely Einstein's theory should be considered as the well-done approximation for the speeds less than the speed of light, whereas for the objects travelling faster than light, Special Relativity should be complemented throughout new arguments, perhaps coined on the basis of this theory. Einstein's postulates define Special Relativity, and in themselves are not the limits to Nature and physics, and, therefore, the sceptics should discuss new models rather than internal consistency of Special Relativity.

In this paper, we present the model of faster-than-light motion which is a compromise with Einstein's Special Relativity, and consistently and constructively describes superluminal particles. Moreover, paradoxically, we show that superluminally traveling particles can be efficiently theorized with making use of Special Relativity, but complemented throughout the additional arguments. In this manner, although in this model Einstein's postulates are broken, the mathematical structure of Minkowski's energy-momentum space remains unchanged, in favour of the significant modifcation to the celebrated Einstein's energy-momentum relation $E^{2}=m^{2} c^{4}+p^{2} c^{2}$ expressing the equivalence principle between mass and energy of a moving particle. Furthermore, the model is constructed in the way such that the invariance with respect to the Poincarfie symmetry, and its special case known as the Lorentz symmetry, which is also present as an internal characteristics of Special Relativity, remains preserved. For the consistency of the model with Einstein's Special Relativity, we propose to take into account the nonparallelism hypothesis, which violates the parallelism between momentum and velocity vectors of a moving particle, in particular present in both Newton's and Einstein's theories of motion. Moreover, the deformation of Einstein's equivalence principle is derived ab initio. The most essential change is an introduction of the speed of light and the mass of a particle which are deformed due to both the violation of momentum-velocity parallelism and deformation of the equivalence principle. Particularly, the deformed speed of light can exceed the value of the speed of light c. These assumptions result in the theoretical model of a moving particle which is able to reach superluminal speeds, but the motion described throughout the deformed speed of light and deformed mass of a particle preserves both the Poincarffe as well as Lorentz invariance of the Minkowski energy-momentum space. Additionally, we provide the discussion of the proposed model in the context of the superluminal neutrinos allegedly detected in 2011 by the OPERA Collaboration of CERN, and the analysis demonstrates that in the proposed model exceeding of the speed of light $\mathrm{c}$ and high-speed mass ejection due to the OPERA neutrinos can be consistently explained, without refereing to the mistakes of the measurement process.

\section{Assumptions}

\subsection{Violated Parallelism}

First of all, we propose the non-parallelism hypothesis: for faster-than-light motion the momentum-velocity parallelism is broken. In other words, if $\alpha$ is an angle between the vectors of velocity $v^{i}$ and momentum $p^{i}$, then for superluminal particles $\alpha \neq 0$. In such a situation, making use of the definition of a scalar product, one receives

$$
\mathrm{v}^{\mathrm{i}} \mathrm{p}_{i}=\mathrm{vp} \cos \alpha
$$

where $v=\sqrt{v^{i} v_{i}}$ is the speed of a particle and $p=\sqrt{p^{i} p_{i}}$ is the value ofthe momentum vector. Making use of the standard definition of the velocity vector which, in particular, is valid for both Einstein's Special Relativity and Newton's mechanics

$$
v_{i}=\frac{\partial E}{\partial p_{i}},
$$

where $\mathrm{E}$ is an energy of a particle, one obtains 


$$
\frac{\partial E}{\partial p}=\frac{\partial E}{\partial p_{i}} \frac{\partial p_{i}}{\partial p}=v^{i} \frac{p_{i}}{p}=v \cos \alpha,
$$

and, consequently, the speed of a particle is

$$
v=\frac{1}{\cos \alpha} \frac{\partial E}{\partial p} .
$$

\subsection{Deformed Special Relativity}

According to Einstein's Special Relativity, the motion of a particle which is equipped with a mass m, energy $\mathrm{E}$, momentum $\mathrm{p}_{\mathrm{i}}$ is described through to the following energy-momentum relation

$$
E^{2}=m^{2} c^{4}+p^{2} c^{2}
$$

where $\mathrm{c}$ is the speed of light, also known as the Einstein equivalence principle or the Hamiltonian constraint of Special Relativity. Both the equivalence principle and absoluteness of the speed of light are the assumptions leading to Special Relativity, called the Einstein postulates. The Einstein theory generalizes the Newton mechanics for the case of the speeds close to c and, moreover, its most essential ramification is the Standard Model of particles and fundamental interactions, which lays the foundations of modern high energy physics, Cf. the Refs. [23-33]. By virtue of Special Relativity, c is a maximal speed which can be reached through a material object in motion and, moreover, the faster-then-light motion corresponds with the negative value of a squared mass of a particle, which in this case is called a tachyon. Let us consider the modified energy-momentum relation

$$
E^{2}=m^{2} c^{4}+p^{2} c^{2}+\Delta(E, p ; m, \alpha),
$$

where a deformation $\Delta$ must be a Lorentz-invariant scalar, and, therefore, a function of even power of p. Because in Special Relativity a deformation is identically vanishing, its presence unambiguously suggests that the deformed equivalence principle will lead to the particle physics beyond the Standard Model. The problem is finding $\Delta$ such that Minkowski's energy-momentum space and both the Poincare and Lorentz symmetries, which lay the structural foundations of Special Relativity and its particular consequence known as the CPT theorem, are valid together with the modified Einstein's postulates.

The fundamental problem is understanding of the deformed equivalence principle (6) from the point of view of the formal context, which in this case is the so-called implicit differentiation well-known from the elementary calculus of many-variable functions, Cf. the Refs. $[34,35,36,37]$. Because $\Delta$ in itself is assumed to be a certain fixed but still unknown function of $\mathrm{E}$ and $\mathrm{p}$, actually the relation (6) defines energy E implicitly as a differentiable function of momentum value $\mathrm{p}$, and, therefore, should be rewritten as the equation

$$
\mathrm{F}(\mathrm{p}, \mathrm{E})=\mathrm{p}^{2} \mathrm{c}^{2}+\mathrm{m}^{2} \mathrm{c}^{4}+\Delta(\mathrm{E}, \mathrm{p} ; \mathrm{m}, \alpha)-\mathrm{E}^{2}=0,
$$

which allows to establish

$$
\begin{aligned}
& F_{p}(p, E)=\frac{\partial F(p, E)}{\partial p}=2 p c^{2}+\frac{\partial \Delta}{\partial p}, \\
& F_{E}(p, E)=\frac{\partial F(p, E)}{\partial E}=-2 E+\frac{\partial \Delta}{\partial E},
\end{aligned}
$$

and, throughout making use of the chain rule for the differentiation of an implicit function of two variables, one has

$$
\frac{\partial E}{\partial p}=\frac{F_{p}(p, E)}{F_{E}(p, E)}=\frac{2 p c^{2}+\frac{\partial \Delta}{\partial p}}{2 E-\frac{\partial \Delta}{\partial E}} .
$$

Consequently, making use of the definition (4), one receives the formula

$$
v=\frac{1}{\cos \alpha} \frac{2 p c^{2}+\frac{\partial \Delta}{\partial p}}{2 E-\frac{\partial \Delta}{\partial E}}
$$

which defines the speed of a moving object according to both the nonparallelism hypothesis (1) and the deformed Special Relativity (6). One sees easily that the superluminal motion, that is $\mathrm{v}>\mathrm{c}$, can be reached whenever

$$
\cos \alpha<\frac{2 p c+\frac{1}{c} \frac{\partial \Delta}{\partial p}}{2 E-\frac{\partial \Delta}{\partial E}},
$$

and, in comparison with the bounds $-1 \leq \cos \alpha \leq 1$.

$$
E \in\left(-\infty,-p c+\frac{1}{2}\left(\frac{\partial \Delta}{\partial E}-\frac{1}{c} \frac{\partial \Delta}{\partial p}\right)\right] \cup\left[p c+\frac{1}{2}\left(\frac{\partial \Delta}{\partial E}+\frac{1}{c} \frac{\partial \Delta}{\partial p}\right), \infty\right) .
$$

\section{Compromise with Special Relativity}

Let us try to establish a deformation $\Delta$ compromising with Special Relativity. In order to preserve the structure of the Minkowski energy-momentum space, we can not modify definitions of energy $\mathrm{E}$ and momentum $\mathrm{p}$ throughout function relations $\widetilde{E}(E)$ and $\tilde{p}(p)$, because this modification changes the metric from a at one to for a curved one and, therefore, the Special Relativity formalism could be broken. However, looking on the derived speed formula (4), one can see a strict analogy with Einstein's Special Relativity when this formula is written in the following way

$$
v=\frac{p \tilde{c}^{2}}{E},
$$

where $\tilde{c}$ is the deformed speed of light

$$
\tilde{c}=\frac{c}{\sqrt{\cos \alpha}} \sqrt{\frac{1+\frac{1}{2 p c^{2}} \frac{\partial \Delta}{\partial p}}{1-\frac{1}{2 E} \frac{\partial \Delta}{\partial E}}},
$$

which, in general, can exceed the speed of light c whenever the condition (12) holds. Applying the formula (15) in the constraint (6), one obtains

$$
E^{2}=\frac{\tilde{m}}{m} p^{2} \tilde{c}^{2}+\tilde{m}^{2} \tilde{c}^{4}+\Delta,
$$

where $\widetilde{m}$ is the deformed mass of a particle 


$$
\tilde{m}=m\left(\frac{\tilde{c}}{c}\right)^{2} \cos ^{2} \alpha .
$$

It is well-visible that in the absence of a deformation, that is $\Delta=0$, the equivalence principle (16) displays a manifestly broken structure of the Minkowski energymomentum space and, therefore, also both Poincare and Lorentz symmetries which lay the foundations of modern particle physics. In order to establish the compromise with Special Relativity, the deformed equivalence principle (16) should be also written in the way of a strict analogy with the Einstein equivalence principle

$$
E^{2}=p^{2} \tilde{c}^{2}+\tilde{m}^{2} \tilde{c}^{4},
$$

for which all properties of Special Relativity are satisfied, but with respect to the deformed speed of light and the deformed mass of a particle. This assumption generates a certain specific form of a deformation $\Delta$, which can be immediately derived

$$
\Delta=\left(1-\frac{\tilde{m}}{m}\right) p^{2} \tilde{c}^{2}=\left(\frac{1-\frac{1}{2 E} \frac{\partial \Delta}{\partial E}}{1+\frac{1}{2 p c^{2}} \frac{\partial \Delta}{\partial p}} \frac{1}{\cos \alpha}-1\right) p^{2} c^{2} .
$$

Consequently, in this state of affairs, the deformed equivalence principle, expressed throughout the nondeformed both speed of light and mass of a particle, has the following form

$$
E^{2}=\frac{1-\frac{1}{2 E} \frac{\partial \Delta}{\partial E}}{1+\frac{1}{2 p c^{2}} \frac{\partial \Delta}{\partial p}} \frac{1}{\cos \alpha} p^{2} c^{2}+m^{2} c^{4}
$$

The relation (20) allows to write

$$
\frac{1+\frac{1}{2 p c^{2}} \frac{\partial \Delta}{\partial p}}{1-\frac{1}{2 E} \frac{\partial \Delta}{\partial E}}=\frac{p^{2} c^{2}}{\cos \alpha} \frac{1}{E^{2}-m^{2} c^{4}}
$$

and, for this reason, the relations (15) and (17) become

$$
\begin{gathered}
\tilde{c}=\frac{p c^{2}}{\cos \alpha} \frac{1}{\sqrt{E^{2}-m^{2} c^{4}}}, \\
\tilde{m}=m \frac{p^{2} c^{2}}{E^{2}-m^{2} c^{4}} .
\end{gathered}
$$

whereas, on the one hand, the speed formula (11) gives

$$
v=\frac{c}{\cos ^{2} \alpha} \frac{(P C)^{3}}{E\left(E^{2}-m^{2} c^{4}\right)},
$$

or, the condition for the angle $\alpha$

$$
\cos ^{2} \alpha=\frac{c}{v} \frac{(p c)^{3}}{E\left(E^{2}-m^{2} c^{4}\right)} .
$$

Equivalently, the relation (24) can be transformed into the depressed cubic equation for energy $\mathrm{E}$

$$
E^{3}-\left(m c^{2}\right)^{2} E-\frac{(p c)^{3}}{\cos ^{2} \alpha} \frac{c}{v}=0,
$$

which by virtue of the Cardano method, Cf. the Refs. $[38,39,40]$, has a real solution

$$
E=P C \frac{\sqrt[3]{1+\sqrt{1-\frac{4 m^{6} c^{4} v^{2}}{27 p^{6}} \cos ^{4} \alpha}}+\sqrt[3]{1-\sqrt{1-\frac{4 m^{6} c^{4} v^{2}}{27 p^{6}} \cos ^{4} \alpha}}}{\sqrt[3]{2 \frac{v}{c} \cos ^{2} \alpha}},
$$

whenever the angle $\alpha$ satisfies the condition

$$
\cos ^{2} \alpha \leq \frac{3 \sqrt{3}}{2}\left(\frac{p}{m c}\right)^{3} \frac{c}{v},
$$

or, after making use of the formula (25),

$$
E^{3}-\left(m c^{2}\right)^{2} E-\frac{2 \sqrt{3}}{9}\left(m c^{2}\right)^{3} \geq 0,
$$

what means that energy of a moving particle must satisfy the bound

$$
E \geq \frac{2 \sqrt{3}}{3} m c^{2} .
$$

In addition, the formula (28) in comparison with the bounds $0 \leq \cos ^{2} \alpha \leq 1$ gives the bound for the speed

$$
v \geq \frac{3 \sqrt{3}}{2} c\left(\frac{p}{m c}\right)^{3}
$$

Moreover, one sees also that $\mathrm{E}>0$ whenever $\frac{4 m^{6} c^{4} v^{2}}{27 p^{6}} \cos ^{4} \alpha>0$, that is the energy (27) is positive for each physical situation.

Interestingly, the relation (24) is able to give a qualitative answer when a particle can exceed the speed of light. Namely, such a situation can took place whenever the following inequality is satisfied

$$
E^{3}-\left(m c^{2}\right)^{2} E-\frac{(p c)^{3}}{\cos ^{2} \alpha}<0,
$$

or, equivalently,

$$
E<\frac{p c}{\cos ^{2 / 3} \alpha}\left(\sqrt[3]{1+\sqrt{1-\frac{4 m^{6} c^{6} \cos ^{4} \alpha}{27 p^{6}}}}+\sqrt[3]{1-\sqrt{1-\frac{4 m^{6} c^{6} \cos ^{4} \alpha}{27 p^{6}}}}\right),
$$

whenever the condition holds

$$
\cos ^{2} \alpha \leq \frac{3 \sqrt{3}}{2}\left(\frac{p}{m c}\right)^{3},
$$

or, after taking into account the fact $0 \leq \cos ^{2} \alpha \leq 1$

$$
p \leq \frac{\sqrt{2} \sqrt[3]{3}}{3} m c .
$$

Alternatively, the inequality (32) can be solved with respect to the angle $\alpha$

$$
\cos ^{2} \alpha<\frac{(p c)^{3}}{E^{3}-\left(m c^{2}\right)^{2} E},
$$


and, throughout the bounds $0 \leq \cos ^{2} \alpha \leq 1$, allows to deduce the following condition for the superluminal motion

$$
p \leq \frac{E}{c} \sqrt[3]{1-\left(\frac{m c^{2}}{E}\right)^{2}}
$$

\section{Eikonal Equation}

Looking on the formula (20), one can see that this relation actually can be transformed into the form

$$
\begin{aligned}
& \frac{1}{E\left(E^{2}-m^{2} c^{4}\right)}\left(2 E-\frac{\partial \Delta}{\partial E}\right) \\
& -\frac{\cos ^{2} \alpha}{(p c)^{3}}\left(2(p c)+\frac{\partial \Delta}{\partial(p c)}\right)=0,
\end{aligned}
$$

which is the first order partial differential equation of eikonal-type, well-known in a whole physics and especially in quantum mechanics and geometrical optics, for the deformation consistent with Special Relativity. After application of the method of separating variables

$$
\Delta(E, p ; m, \alpha)=\Delta_{1}(E ; m, \alpha)+\Delta_{2}(p ; m, \alpha),
$$

the equation (38) becomes

$$
\frac{1}{E\left(E^{2}-m^{2} c^{4}\right)}\left(2 E-\frac{d \Delta_{1}}{d E}\right)=\frac{\cos \alpha}{(p c)^{3}}\left(2(p c)+\frac{d \Delta_{2}}{d(p c)}\right) .(40)
$$

In the equation (40), the left hand side depends only on the variable $\mathrm{E}$, whereas the right hand side depends only on the variable p. Consequently, both these expressions are equal at most a separation constant $A=\frac{1}{\varepsilon^{2}}$, where $\varepsilon$ is the separation energy parameter,

$$
\begin{gathered}
\frac{1}{E\left(E^{2}-m^{2} c^{4}\right)}\left(2 E-\frac{d \Delta_{1}}{d E}\right)=\frac{1}{\varepsilon^{2}}, \\
\frac{\cos \alpha}{(p c)^{3}}\left(2(p c)+\frac{d \Delta_{2}}{d(p c)}\right)=\frac{1}{\varepsilon^{2}} .
\end{gathered}
$$

Consequently, the problem of finding the deformation is given throughout the system of the first order ordinary differential equations

$$
\begin{aligned}
& \frac{d \Delta_{1}}{d E}=-\frac{1}{\varepsilon^{2}} E^{3}+\left(2+\frac{m^{2} c^{4}}{\varepsilon^{2}}\right) E, \\
& \frac{d \Delta_{2}}{d(p c)}=\frac{1}{\varepsilon^{2} \cos \alpha}(p c)^{3}-2(p c),
\end{aligned}
$$

which can be solved through straightforward integration

$$
\begin{gathered}
\Delta_{1}(E, p ; m, a)=-\frac{1}{4 \varepsilon^{2}} E^{4}+\left(1+\frac{m^{2} c^{4}}{2 \varepsilon^{2}}\right) E^{2}+C_{1}(m, a), \\
\Delta_{2}(E, p ; m, a)=-\frac{1}{4 \varepsilon^{2} \cos \alpha}(p c)^{4}-(p c)^{2}+C_{2}(m, a),
\end{gathered}
$$

where $C_{1}(m, a)$ and $C_{2}(m, a)$ are integration onstants, and, therefore,

$$
\begin{aligned}
& \Delta(E, p ; m, a)=-\frac{1}{4 \varepsilon^{2}} E^{4}+\left(1+\frac{m^{2} c^{4}}{2 \varepsilon^{2}}\right) E^{2} \\
& +\frac{1}{4 \varepsilon^{2} \cos \alpha}(p c)^{4}-(p c)^{2}+\Delta_{0}(m, a),
\end{aligned}
$$

where $\Delta_{0}(m, a)=C_{1}(m, a)+C_{2}(m, a)$ is a cumulative integration constant.

Nevertheless, implementation of the solution (47) in the deformed equivalence principle (6) gives the relation

$$
\frac{1}{4 \varepsilon^{2}} E^{4}-\frac{m^{2} c^{4}}{2 \varepsilon^{2}} E^{2}-\frac{1}{4 \varepsilon^{2} \cos \alpha}(p c)^{4}-m^{2} c^{4}-\Delta_{0}(m, a)=0,(48)
$$

which can be interpreted as the algebraic equation for squared energy $\mathrm{E}^{2}>0$

$$
E^{4}-2\left(m c^{2}\right)^{2} E^{2}-\left[\frac{(p c)^{4}}{\cos \alpha}+4 \varepsilon^{2}\left(m^{2} c^{4}+\Delta_{0}(m, a)\right)\right]=0,(49)
$$

which can be solved immediately

$$
E^{2}=m^{2} c^{4}+m^{2} c^{4} \sqrt{1+\frac{1}{\cos \alpha}\left(\frac{p}{m c}\right)^{4}}
$$

and, after comparison with (6), leads to the following form of the deformation

$$
\Delta=-p^{2} c^{2}+m^{2} c^{4} \sqrt{\begin{array}{l}
1+\frac{1}{\cos \alpha}\left(\frac{p}{m c}\right)^{4} \\
+\left(\frac{2 \varepsilon}{m c^{2}}\right)^{2}\left(1+\frac{\Delta_{0}(m, \alpha)}{\left(m c^{2}\right)^{2}}\right)
\end{array}}
$$

Taking into account the following initial condition

$$
\Delta(0,0 ; m, a)=\Delta_{0}(m, a),
$$

the formula (51) gives the equation

$$
\Delta_{0}^{2}-4 \varepsilon^{2} \Delta_{0}-\left(m c^{2}\right)^{2}\left(4 \varepsilon^{2}+\left(m c^{2}\right)^{2}\right)=0,
$$

which has two solutions

$$
\Delta_{0}(m, a)=\left\{4 \varepsilon^{2}+\left(m c^{2}\right)^{2},-\left(m c^{2}\right)^{2}\right\} .
$$

However, according to the constraint (6), the initial condition (52) gives

$$
\Delta_{0}=-m^{2} c^{4},
$$

and, for this reason, one obtains

$$
\varepsilon^{2}=-\frac{1}{2}\left(m c^{2}\right)^{2}
$$

In this manner, the deformation consistent with Special Relativity is

$$
\Delta=-p^{2} c^{2}+m^{2} c^{4} \sqrt{1+\frac{1}{\cos \alpha}\left(\frac{p}{m c}\right)^{4}},
$$

and, consequently, the deformed energy-momentum relation is

$$
E^{2}=m^{2} c^{4}+m^{2} c^{4} \sqrt{1+\frac{1}{\cos \alpha}\left(\frac{p}{m c}\right)^{4}} .
$$


Interestingly, alternatively the eikonal equation (38) can be written in the form

$$
\begin{aligned}
& \frac{1}{E\left(E^{2}-m^{2} c^{4}\right) \cos \alpha}\left(2 E-\frac{\partial \Delta}{\partial E}\right) \\
& -\frac{1}{(p c)^{3}}\left(2(p c)+\frac{\partial \Delta}{\partial(p c)}\right)=0,
\end{aligned}
$$

which, throughout making use of the separation of variables (39), leads to slightly different system of first order ordinary differential equations

$$
\begin{gathered}
\frac{d \Delta_{1}}{d E}=-\frac{\cos \alpha}{\tilde{\varepsilon}^{2}} E^{3}+\left(2+\frac{m^{2} c^{4}}{\tilde{\varepsilon}^{2}} \cos \alpha\right) E, \\
\frac{d \Delta_{2}}{d(p c)}=\frac{1}{\tilde{\varepsilon}^{2}}(p c)^{3}-2(p c),
\end{gathered}
$$

where we have considered a new separation constant $\widetilde{A}=\frac{1}{\tilde{\varepsilon}^{2}}$, which results in the solution

$$
\begin{aligned}
& \Delta(E, p ; m, a)=-\frac{\cos \alpha}{4 \tilde{\varepsilon}^{2}} E^{4}+\left(1+\frac{m^{2} c^{4}}{2 \tilde{\varepsilon}^{2}} \cos \alpha\right) E^{2} \\
& +\frac{1}{4 \tilde{\varepsilon}^{2}}(p c)^{4}-(p c)^{2}+\widetilde{\Delta}_{0},
\end{aligned}
$$

where $\widetilde{\Delta}_{0}$ is a cumulative integration constant, which applied in the constraint (6) leads to the following constraint

$$
E^{4}-2\left(m c^{2}\right)^{2} E^{2}-\left[\begin{array}{l}
\frac{(p c)^{4}}{\cos \alpha} \\
+\frac{4 \tilde{\varepsilon}^{2}}{\cos \alpha}\left(m^{2} c^{4}+\tilde{\Delta}_{0}(m, a)\right)
\end{array}\right]=0 \text { (63) }
$$

whose positive solution is

$$
E^{2}=m^{2} c^{4}+m^{2} c^{4} \sqrt{1+\frac{1}{\cos \alpha}\left(\frac{p}{m c}\right)^{4}}
$$

and, therefore,

$$
\Delta=-p^{2} c^{2}+m^{2} c^{4} \sqrt{1+\frac{1}{\cos \alpha}\left(\frac{p}{m c}\right)^{4}}
$$

Nevertheless, taking into account both the initial condition (52) for the solution (65), one obtains

$$
\begin{gathered}
\tilde{\Delta}_{0}=\Delta_{0}=-\left(m c^{2}\right)^{2}, \\
\tilde{\varepsilon}^{2}=\varepsilon^{2} \cos \alpha=-\frac{1}{2}\left(m c^{2}\right)^{2} \cos \alpha,
\end{gathered}
$$

and, moreover, exactly the already established results (57) and (58). Therefore, the solutions (47) and (62) are fully compatible each to other.
Taking into account the result (58), the relations (22), (23), and (24), can be expressed throughout the momentum value

$$
\begin{gathered}
\tilde{c}=c \frac{\frac{1}{\cos \alpha} \frac{p}{m c}}{\left(1+\frac{1}{\cos \alpha}\left(\frac{p}{m c}\right)^{4}\right)^{1 / 4}}, \\
\tilde{m}=m \frac{\frac{1}{\cos \alpha}\left(\frac{p}{m c}\right)^{2}}{1+\frac{1}{\cos \alpha}\left(\frac{p}{m c}\right)^{4}}, \\
v=c \frac{\frac{1}{\cos \alpha}\left(\frac{p}{m c}\right)^{3}}{\sqrt{1+\frac{1}{\cos \alpha}\left(\frac{p}{m c}\right)^{4}+\left(1+\frac{1}{\cos \alpha}\left(\frac{p}{m c}\right)^{4}\right)^{3 / 2}}}
\end{gathered}
$$

or, throughout energy

$$
\begin{gathered}
\tilde{c}=\frac{c}{\cos ^{3 / 4} \alpha} \frac{E}{m c^{2}} \sqrt{\frac{E^{2}-2 m^{2} c^{4}}{E^{2}-m^{2} c^{4}}}, \\
\tilde{m}=m \sqrt{\cos \alpha} \frac{E^{2}}{m^{2} c^{4}} \frac{E^{2}-2 m^{2} c^{4}}{E^{2}-m^{2} c^{4}}, \\
v=\frac{c}{\cos ^{5 / 4} \alpha} \frac{E^{2}}{\left(m c^{2}\right)^{3}} \frac{\left(E^{2}-2 m^{2} c^{4}\right)^{3 / 2}}{E^{2}-m^{2} c^{4}} .
\end{gathered}
$$

\section{Lorentz Invariance}

Special Relativity with its mathematical structure given by the Minkowski energy-momentum space, possesses the remarkable invariance with respect to action of the Lorentz transformation, Cf. the Refs. [41-51]. In the language of quantum field theory, which is a particularly important result of Special Relativity and lays the foundations of modern particle physics, nuclear physics, and astrophysics, the Lorentz violation is equivalent to violation of the CPT symmetry, that is invariance with respect to simultaneous action of charge conjugation, parity transformation, and time reversal, and under the CPT theorem all physical phenomena must be CPT invariant. However, the CP violation has already been detected experimentally, studied throughout the recent years, and its various consequences are one of the most intensively developing trends of modern theoretical physics, Cf. Refs. [52-67].

Since the equivalence principle (18) does not change definitions of $\mathrm{E}$ and $\mathrm{p}$ and preserves the metric structure of the Minkowski energy-momentum space, this leads to a straightforward analogy with the Einstein theory, and, consequently, should define a Lorentz invariant theory. Considering the deformed energy-momentum four-vector

$$
\tilde{p}^{u}=\left[E, p^{i} \tilde{c}\right],
$$

one can construct the energy-momentum interval 


$$
\tilde{s}^{2}=\eta_{\mu v} \widetilde{p}^{u} \widetilde{p}^{v}=-E^{2}+p^{2} \tilde{c}^{2}=-\tilde{m}^{2} \tilde{c}^{4},
$$

Where $\eta_{\mu v}=\operatorname{diag}(-1,1,1,1)$ is the Minkowski metric satisfying the orthogonality condition $\eta^{\lambda \mu} \eta_{\mu v}=\delta_{v}^{\lambda}$. For this energy-momentum space we should impose ad hoc invariance with respect to the Lorentz transformation

$$
\tilde{p}^{\prime} \mu \Lambda_{v}^{\mu} \widetilde{p}^{v}
$$

Where $\Lambda_{v}^{\mu}$ are the Lorentz matrices. The Lorentz invariance

$$
\tilde{s}^{\prime 2}=\eta_{\mu v} \tilde{p}^{\prime} u \widetilde{p}^{\prime v}=\tilde{s}^{2}
$$

with the standard rules of the Lorentz transformation

$$
\begin{gathered}
\eta_{\mu v} \Lambda_{k}^{\mu} \Lambda_{\lambda}^{v}=\eta_{k \lambda}, \\
\Lambda^{\alpha \mu} \Lambda_{\lambda}^{v}=\eta_{\lambda}^{\alpha} \eta^{\mu v}, \\
\Lambda^{\alpha \mu} \Lambda_{\alpha}^{v}=\eta^{\mu v},
\end{gathered}
$$

is present in the deformed relativity, if and only if one considers the deformed speed of light $\tilde{c}$ and the deformed mass of a particle $\widetilde{m}$ as a constant parameters. Then, the light cone, which in Special relativity defines the limits for the superluminal motion, is also deformed since this is determined with respect to $\tilde{c}$, instead of the speed of light c, which can exceed the value of c. Otherwise, one has to deal with the Lorentz violation.

Application of the formulas (58), (68) and (69), within the equivalence principle (18) or the interval (75) leads to the non-trivial physical values of momentum p defined by the algebraic equation

$$
A\left(\frac{p}{m c}\right)^{16}+B\left(\frac{p}{m c}\right)^{12}+C\left(\frac{p}{m c}\right)^{8}+D\left(\frac{p}{m c}\right)^{4}+E=0
$$

which is a quartic equation in $\left(\frac{p}{m c}\right)^{4} \geq 0$, wherein

$$
\begin{gathered}
A=(1-\cos \alpha)^{2} \cos ^{5} \alpha, \\
B=4 \cos ^{8} \alpha-8 \cos ^{7} \alpha+3 \cos ^{6} \alpha+2 \cos ^{4} \alpha-1, \\
C=\left(6 \cos ^{4} \alpha-12 \cos ^{3} \alpha+3 \cos ^{2} \alpha+4\right) \cos ^{5} \alpha, \\
D=\left(4 \cos ^{4} \alpha-8 \cos ^{3} \alpha+\cos ^{2} \alpha+2\right) \cos ^{6} \alpha, \\
E=(2-\cos \alpha) \cos ^{10} \alpha .
\end{gathered}
$$

Its solutions can be presented in the form

$$
\begin{aligned}
& p_{1,2}=m c\left(-\frac{B}{4 A}-\Gamma \pm \sqrt{-\Gamma^{2}-p+\frac{q}{\Gamma}}\right)^{1 / 4}, \\
& p_{3,4}=m c\left(-\frac{B}{4 A}+\Gamma \pm \sqrt{-\Gamma^{2}-p-\frac{q}{\Gamma}}\right)^{1 / 4}
\end{aligned}
$$

where

$$
\begin{gathered}
p=\frac{C}{2 A}-\frac{3}{16}\left(\frac{B}{A}\right)^{2}, \\
q=\frac{1}{32}\left(\frac{B}{A}\right)^{3}-\frac{B C}{8 A^{2}}+\frac{D}{4 A}, \\
\Gamma=\frac{1}{2 \sqrt{3 A}} \sqrt{-4 A p+\Upsilon+\frac{C^{2}-3 B D+12 A E}{\Upsilon}} \\
\Upsilon=\left(\delta+\sqrt{\delta^{2}-\left(C^{2}-3 B D+12 A E\right)^{3}}\right)^{1 / 3}, \\
\delta=\frac{1}{2} C\left(2 C^{2}-9 B D-72 A E\right)+27\left(A D^{2}+B^{2} E\right) .
\end{gathered}
$$

This is clear that in the presented model a particle motion is both explicitly and entirely expressed throughout the free parameters of the theory

$$
\begin{gathered}
p=f(\cos \alpha) m c, \\
E=m c^{2} \sqrt{1+\sqrt{1+\frac{f^{4}(\cos \alpha)}{\cos \alpha}},},
\end{gathered}
$$

where $f(x)$ is a certain smooth function. In the light of this state of affairs, the formulas (68) and (69) show that the deformed speed of light $\tilde{c}$ and the deformed mass of a particle $\tilde{m}$ are also the parameters of the theory.

In this manner, the Lorentz invariance of the model of deformed relativity, considered in this paper, has been proven.

\section{Discussion}

In the CERN experiments, the measurements due to the detectors of the OPERA Collaboration, Cf. the Refs. [1,2], had allowed to establish the following faster-than-light speed of neutrinos

$$
\frac{v}{c}=1.0000248(28) .
$$

It is worth stressing that, at the present level of both the formal development of theoretical physics and the mind of majority of physicists this measurement result is impossible to be explained constructively and consistently within the commonly believed standard theoretical approaches and frameworks offered throughout modern high energy physics. Nevertheless, such an elucidation of the superluminal neutrinos can be performed throughout making use of the model of deformed relativity presented in this paper. Accepting the state of affairs propagated through the Standard Model, wherein the neutrino is considered as massless, according to the aforementioned formulas (68), (69) and (70), for the case of the OPERA neutrinos

$$
\begin{gathered}
\tilde{m}=0 \mathrm{~kg}, \\
\cos \alpha=\frac{1}{(v / c)^{4}}=0.9999006(94), \\
\tilde{c}=\frac{c}{\cos ^{3 / 4} \alpha}=2.997701294 \cdot 10^{8} \frac{\mathrm{m}}{\mathrm{s}^{2}},
\end{gathered}
$$


and precisely the deforming angle is

$$
\alpha=0.8074741(17)^{\circ}
$$

Furthermore, in general, the presented theoretical model offers much more than it was already written here. Namely, the presence of a non-trivial deformation in the equivalence principle can be understood as the description of a measurement process of a particle motion. Then, all information about the possible mistakes could be contained in such a deformation. However, this is merely an interpretation of this model of physical reality which, from an objective point of view, is first of all able to explain the measured parameters of a particle motion throughout using a rational analysis which is consistent with the well-established knowledge of Special Relativity. It should be emphasized that at the modern-day experimental level, nobody really knows what actually happens with particles at high energies and, particularly, what kind of processes affect the measurement process. In the presented scenario all possible reasons are hidden in a deformation of the equivalence principle due to the violence of momentum-velocity parallelism. We hope for further developments in this physical theory and philosophy.

\section{References}

[1] OPERA Collaboration (2011). Measurement of the neutrino velocity with the OPERA detector in the CNGS beam. arXiv: $1109.4897 \mathrm{v} 1$.

[2] CERN (2011). OPERA experiment reports anomaly in ight time of neutrinos from CERN to Gran Sasso. September 23, 2011.

[3] Cartlidge, E. (2012). Breaking News: Error Undoes Faster-ThanLight Neutrino Results. Science Insider, February 22, 2012.

[4] Reich, E.S. (2012). Flaws found in faster-than-light neutrino measure- ment. Nature News.

[5] ICARUS Collaboration (2012). Measurement of the neutrino velocity with the ICARUS detector at the CNGS beam. arXiv: 1203. 3433, Phys. Lett. B 713 (1), 17-22.

[6] CERN (2012). Neutrinos sent from CERN to Gran Sasso respect the cosmic speed limit. June 8, 2012.

[7] Bilaniuk, O.M.P., Deshpande, V.K. \& Sudarshan, E.C.G. (1962). "Meta" Relativity. Amer. J. Phys. 30, 718-723.

[8] Feinberg, G. (1967). Possibility of Faster-Than-Light Particles. Phys. Rev. 159(5), 1089-1105.

[9] Sudarshan, E.C.G. (1968). Theory of Particles Travelling FasterThan- Light. Syracuse University Report.

[10] Sudarshan, E.C.G. (1968). The Nature of Faster-Than-Light Particles and Their Interactions. Delhi University Report.

[11] Bilaniuk, O.M., Brown, S.L., DeWitt, B., Necomb, W.A., Sachs, M., Sudarshan, E.C.G. \& Yoshikawa, S. (1969). More About Tachyones. Physics Today 22 (12), 47-52.

[12] Bilaniuk, O.M. \& Sudarshan, E.C.G. (1969). Particles Beyond the Light Barrier. Physics Today 22 (5), 43-51.

[13] Camenzind, M. (1970). Relativity and Free Tachyons. Gen. Rel. Grav. 1 (1), 41-62.

[14] Csonka, P.L. (1970). Causality and Faster than Light Particles. Nucl. Phys. B21, 436-444.

[15] Feinberg, G. (1970). Particles that go faster than light. Scientific American 222 (2), 69-76.

[16] Fitzgerald, P. (1970). Tachyons, Backwards Causation and Freedom. Boston Studies in the Philosophy of Science 8, 415-436.

[17] Newton, R.G. (1970). Particles that Travel Faster than Light?. Science 167, 1659-1574.

[18] Fitzgerald, P. (1974). On Retrocausality. Philosophia 4, 513-551.

[19] Kirzhnitz, D.A. \& Sazonov, V.N. (1974). Superluminal Motions and Special Relativity Theory (in Russian), in Einsteinovskii Sbornik 1973 ed. by V.L. Ginzburg, Nauka, Moscow, USSR, pp. 84-111.
[20] Stinner, A. \& Winchester, I. (1981). The physics of Star Trek. New Scientist 24 (3), 884-887.

[21] Chapman, T. (1982). Time: Philosophical Analysis. Springer, New York, USA.

[22] Fayngold, M. (2002). Special Relativity and Motions Faster than Light. Wiley-VCH, Weinheim, Germany.

[23] Scheck, F. (2012). Classical Field Theory. On Electrodynamics, Non- Abelian Gauge Theories and Gravitation, Springer, BerlinHeidelberg, Germany.

[24] mNagashima, Y. (2010). Elementary Particle Physics.Volume 1: Quan- tum Field Theory and Particles, Wiley-VCH, Weinheim, Germany.

[25] Huang. K (2007). Fundamental Forces of Nature: The Story of Gauge Fields. World Scientific, Singapore.

[26] Wiedemann, H. (2007). Particle Accelerator Physics. 3rd ed., Springer, Berlin-Heidelberg, Germany.

[27] Zeidler, E. (2006). Quantum Field Theory I: Basics in Mathematics and Physics. A Bridge between Mathematicians and Physicists. Springer, Berlin-Heidelberg, Germany.

[28] Guidry, M. (2004). Gauge Field Theories: An Introduction with Appli- cations. Wiley-VCH, Weinheim, Germany.

[29] Sexl, R.U. \& Urbantke, H.K. (2001). Relativity, Groups, Particles: Spe- cial Relativity and Relativistic Symmetry in Field and Particle Physics. Springer, Wien, Austria.

[30] Perkins, D.H. (2000). Introduction to High Energy Physics. Cambridge University Press, Cambridge, UK.

[31] Martin, B.R. \& Shaw, G. (1997). Particle Physics. 2nd ed., John Wiley \& Sons, Chichester, UK.

[32] Weinberg, S. (1995). The Quantum Theory of Fields. Vol. I Foundations. Cambridge University Press, Cambridge, UK. Rosser, W.G.V. (1969). Relativity and High Energy Physics. Wykeham Publications, London, UK.

[33] Hagedorn, R. (1964). Relativistic Kinematics: A Guide to Kinematic Problems of High Energy Physics. W.A. Benjamin, New York, USA.

[34] Larson, R. \& Edwards, B.H. (2010). Calculus, Ninth Edition. Brooks/Cole, Cengage Learning, Belmont, USA

[35] Giaquinta, M. \& Modica, G. (2009). Mathematical Analysis: An Intro- duction to Functions of Several Variables. Birkhauser, New York, USA.

[36] Kaplan, W. (2003). Advanced Calculus. 5th ed., Pearson Education, New Jersey, USA.

[37] Cheney, W. (2001). Analysis for Applied Mathematics. Springer, New York, USA.

[38] Grillet, P.A. (2007). Abstract Algebra, 2nd ed., Springer, New York, USA.

[39] Vinberg, E.B. (2003). A Course in Algebra. American Mathematical Society, Providence, USA.

[40] Tignol, J.-P. (2001). Galois' Theory of Algebraic Equations. World Scientific, Singapore.

[41] Einstein, A. (1921). Relativity. The Special and General Theory. Henry Holt and Company, New York, USA.

[42] Lorentz, H.A., Einstein, A., Minkowski, H. \& Weyl, H. (1952) The Principle of Relativity: A Collection of Original Memoirs on the Special and General Theory of Relativity. Dover, USA.

[43] Pauli, W. (1958). Theory of Relativity. Pergamon Press, London, UK.

[44] Fock, V. (1959). The Theory of Space Time and Gravitation. Pergamon Press, London, UK.

[45] Weinberg, S. (1972). Gravitation and Cosmology: Principles and Appli- cations of the General Theory of Relativity. John Wiley \& Sons, New York, USA.

[46] D'Inverno, R. (1998). Introducing Einstein's Relativity. Oxford University Press, Oxford, UK.

[47] Schwarz, P.M. \& Schwarz, J.H. (2004). Special Relativity: From Einstein to Strings. Cambridge University Press, Cambridge, UK.

[48] Stephani, H. (2004). Relativity: An Introduction to Special and General Relativity. 3rd ed., Cambridge University Press, Cambridge, UK

[49] Ehlers, J. \& L ammerzahl, C. (Eds.) (2006). Special Relativity. Will it Survive the Next 101 Years?. Springer, Berlin-Heidelberg, Germany.

[50] Rindler, W. (2006). Relativity: Special, General and Cosmological $2^{\text {nd }}$ ed., Oxford University Press, New York, USA.

[51] Ferraro, R. (2007). Einstein'S Space-Time: An Introduction to Special and General Relativity. Springer, New York, USA. 
[52] Kobayashi, M. (2009). CP Violation and Flavor Mixing. Prog. Theor. Phys. 122 (1), 1-13.

[53] Rev. Mod. Phys. 81, 1019-1025.

[54] Maskawa, T. (2009).What does CP violation tell us?. Prog. Theor. Phys. 122 (1), 15-21.

[55] Rev. Mod. Phys. 81, 1027-1030.

[56] Beyer, M. (Ed.) (2002). CP Violation in Particle, Nuclear and Astro- physics. Springer, Berlin-Heidelberg, Germany.

[57] Bigi, I.I. \& Sanda, A.I. (2009). CP Violation. 2nd ed., Cambridge University Press, Cambridge, UK.

[58] Branco, G.C., Lavoura, L. \& Silva, J.P. (1999). CP Violation. Oxford University Press, New York, USA.

[59] Ginzburg, I.F. \& Krawczyk, M. (2005). Symmetries of two Higgs doublet model and CP violation. Phys. Rev. D 72, 115013.

[60] Glinka, L.A. (2012). thereal Multiverse: A New Unifying Theoretical Approach to Cosmology, Particle Physics, and Quantum Gravity, Cambridge International Science Publishing, Great Abington, UK.
[61] Glinka, L.A. (2010). CP violation, massive neutrinos, and its chiral con- densate: new results from Snyder noncommutative geometry. Apeiron 17 (4), 223-242.

[62] Kleinknecht, K. (2003). Uncovering CP Violation: Experimental Clarication in the Neutral K Meson and B Meson Systems. Springer, Berlin- Heidelberg, Germany.

[63] Koranga, B.S. (2011). CP Violation in Neutrino Oscillation Due to Planck Scale E_ects. Int. J. Theor. Phys. 50 (1), 35-41.

[64] Leader, E. \& Predazzi, E. (1996). An Introduction to Gauge Theories and Modern Particle Physics, Volume 2: CP-violation, QCD and hard processes. Cambridge University Press, Cambridge, UK.

[65] Maccione, L., Taylor, A.M., Mattingly, D.M. \& Liberati, S. (2009). Planck-scale Lorentz violation constrained by Ultra-High-Energy CosmicRays. JCAP 04, 022.

[66] Sanda, A.I. (2009). The Road to the Discovery of Large CP Violation inB Decays. Prog. Theor. Phys. 122 (1), 103-123.

[67] Sozzi, M.S. (2008). Discrete Symmetries and CP Violation: From Ex-periment to Theory. Oxford University Press, New York, USA. 